25 Research Square

\title{
Delirium During COVID-19 Pandemic: Were We Over-Reporting an Overlooked Syndrome?
}

Júlio César Garcia Alencar ( $\square$ julio.alencar@hc.fm.usp.br)

Hospital das Clínicas da Faculdade de Medicina da Universidade de São Paulo

Flávia Barreto Garcez Carvalho

Hospital das Clínicas da Faculdade de Medicina da Universidade de São Paulo

Alicia Duddy Müller Veiga

Hospital das Clínicas da Faculdade de Medicina da Universidade de São Paulo

Gabriela Souza Stanzani

Hospital das Clínicas da Faculdade de Medicina da Universidade de São Paulo

lan Ward Abdalla Maia

Hospital das Clínicas da Faculdade de Medicina da Universidade de São Paulo

Isabella Hiray Pera

Hospital das Clínicas da Faculdade de Medicina da Universidade de São Paulo

Guilherme Tetsuo Yokoy Numakura

Hospital das Clínicas da Faculdade de Medicina da Universidade de São Paulo

Leticia Ruivo Santos

Hospital das Clínicas da Faculdade de Medicina da Universidade de São Paulo

Thiago Junqueira Avelino Silva

Hospital das Clínicas da Faculdade de Medicina da Universidade de São Paulo

Heraldo Possolo Souza

Hospital das Clínicas da Faculdade de Medicina da Universidade de São Paulo

\section{Research Article}

Keywords: Emergency, Delirium, Elderly,

Posted Date: November 17th, 2021

DOI: https://doi.org/10.21203/rs.3.rs-1058910/v1

License: (1) This work is licensed under a Creative Commons Attribution 4.0 International License.

Read Full License 


\section{Abstract \\ Background}

Delirium is an overlooked syndrome in Emergency Departments (ED) around the world. Previous studies demonstrated high incidence of Delirium in elderly patients with COVID-19, however, these studies did not compare Delirium incidences in patients admitted for SARS-CoV-2 infection or sepsis for other etiologies.

\section{Methods}

This is an observational study that analyzed elderly patients ( $>65$ years) from two cohort studies that recruited patients in the ED at Hospital Clínicas da Faculdade de Medicina da Universidade de São Paulo, an urban, academic department in São Paulo, Brazil. The first cohort studied patients with sepsis and was conducted between September 30, 2019, and March 17, 2020. The second cohort studied COVID-19 patients between April 8, 2021, and May 28, 2021. Our primary outcome was incidence of delirium at admission in ED, which we defined using the Confusion Assessment Method (CAM). Additional study information was collected from medical records.

\section{Results}

We included 141 patients in our final analysis, with a median age of 72 (IQR 68-79) years and a predominance of the male sex (58\%). We identified delirium at admission in 31 participants (22\%), 20 in septic group and 11 in COVID-19 group $(p=0.70)$.

\section{Conclusion}

This present study does not support that elderly patients with COVID-19 have higher risk for Delirium at admission in Emergency Department when compared to patients hospitalized with sepsis for other etiologies. In spite of sepsis for COVID-19 have had more than 28\% mortality, COVID-19 was not associated with higher mortality in elderly than sepsis for other etiologies.

\section{Background}

Delirium is a neuropsychiatric emergency characterized by disturbance in attention and awareness. (1) Delirium is common, life-threatening, and often a preventable cause of morbidity among older patients who presents to the emergency department (ED). (1) (2) Delirium indicates acute and severe brain dysfunction, and it is associated with worse outcomes, including increased length of stay, Intensive Care Unit (ICU) admission, persistent cognitive decline, and mortality. (3) 
The incidence of delirium in elderly patients admitted to the ED before COVID-19 ranges from 7 to $24 \%$. (2) Despite this high prevalence, emergency physicians and nurses do not routinely evaluate ED patients for delirium and miss this important and deadly syndrome in $70 \%$ of cases. (3) (4)

The impact of the SARS-CoV-2 infection on delirium incidence is not yet fully clarified. Neurological symptoms are the most common extrapulmonary manifestation in COVID-19 patients, present in 35-50\% of hospitalized in wards and up to $70 \%$ of patients admitted to the ICU. (5)(6)(7) Moreover, studies have shown a higher incidence of delirium in elderly patients with COVID-19 admitted at ED, attributed to inflammation, hypoxemia, thrombosis, sedation, social isolation, and a possible neurotropism of SARSCoV-2. (8)(9)

However, it is not known whether COVID-19 patients present a higher incidence of delirium when compared to other infectious diseases. Therefore, we hypothesized that, since the SARS-CoV-2 may induce neurologic injury by itself, elderly patients hospitalized with COVID-19 should have a higher incidence of delirium than patients hospitalized with sepsis by other etiologies. Furthermore, this essay critically examines if delirium in the Emergency Department is a marker of mortality during hospitalization.

\section{Methods}

\subsection{Aim, design and setting}

This is an observational study that analyzed and compared Delirium incidences in elderly patients with COVID-19 and sepsis for other etiologies from two cohorts which recruited patients in the emergency department at Hospital das Clínicas da Faculdade de Medicina da Universidade de São Paulo (HCFMUSP), São Paulo, Brazil.

Our primary outcome was the incidence of delirium at admission in ED, which we defined using the Confusion Assessment Method (CAM). $(12,13)$ Secondary outcomes were ICU admission and in-hospital death.

Admissions to our ED are centrally managed by the Regulatory Central of the State of São Paulo, and severely ill patients are preferably referred to the hospital. During the COVID-19 pandemic, HCFMUSP became a major center for COVID-19 treatment in São Paulo, the epicenter of the pandemic in Brazil. In March 2020, the main hospital building was converted to a COVID-19-only facility, dedicating 1000 beds to the care of infected patients.

We adhered to the STROBE guidelines.

\subsection{Selection of Participants}

The first cohort study, conducted between September 30, 2019, and March 17, 2020, before the first COVID-19 case was diagnosed on our hospital, and analyzed the association between delirium and brain 
injury biomarkers in elderly septic patients admitted to the ED. Elderly patients ( $>65$ years) admitted less than 24 hours for sepsis were eligible to participate. Sepsis was defined as confirmed infection and an increase of 2 points or more in the Sequential Organ Failure Assessment (SOFA). (10) Patients in exclusive palliative care or previously hospitalization in the last 30 days were excluded. The protocol for this study was approved by the local Ethics Committee in Research (Protocol number 77169716.2.0000.0068), and informed consent by writing was obtained from all patients or legal guardians prior to inclusion.

The second cohort included all elderly patients admitted less than 24 hours for COVID-19 from April 8, 2021, to May 28, 2021. We excluded palliative care patients, previous hospitalization in the last 30 days, and the absence of SARS-CoV-2 confirmed infection. Confirmation of infection by SARS-CoV-2 was performed by real-time polymerase chain reaction (RT-PCR) in nasopharyngeal smear or tracheal aspirate. The study protocol was approved by the local Research Ethics Committee (protocol number 30417520.0.0000.0068). Written informed consent or verbal authorization from the patient or responsible family member was documented in the medical record to guarantee the patient's anonymity preservation.

We adhered to the principles expressed in the Declaration of Helsinki. All patient-identifiable information was stored in secure electronic servers, with access restricted to our researchers.

\subsection{Measurements}

Trained medical and nurses prospectively performed structured interviews with patients and their parents at admission, assessing all patients in the ED with age $>65$ years who met the inclusion criteria for Delirium through the Confusion Assessment Method (CAM). Daily additional study information was collected by reviewing electronic medical records, nursing records, consulting notes, laboratory tests, and radiologic examinations from the complete hospitalizations. Completed standardized electronic case report forms using Research Electronic Data Capture resources. (11)

We collected data on demographics (age, sex, ethnicity, and literacy), clinical history (previous diagnoses and medications, physical examination, and supplemental oxygen), premorbid functional status (Katz Activities of Daily Living Scale), and laboratory tests routinely collected on admission (complete blood cell count, D-dimer, C-reactive protein, urea, creatinine, and albumin). We defined the first available results from within the first 24 hours of hospitalization as the admission results of laboratory tests.

\subsection{Analysis}

Considering that the incidence of delirium in elderly patients admitted to the ED for COVID-19 or other causes was 28 and $7 \%$, respectively $(2,8)$, we calculated a sample of 100 patients to demonstrate that SARS-CoV-2 infection is four times more likely to develop Delirium than sepsis with $80 \%$ power. When we started the COVID-19 cohort study, we had already recruited 95 patients in the Sepsis cohort study.

We expected difficulties in recruiting patients for two reasons: 1 . Our center is reference for severe cases of COVID-19, and most of the patients were admitted after 24 hours of admission in other hospitals; 2. 
Immunization against SARS-CoV-2 started in São Paulo in January 2021 and had already reduced the number of elderly patients hospitalized in our center.

We reported descriptive results for the total sample, comparing the variables of interest according to the overall incidence of delirium. We used the chi-square test to compare categorical variables, and we used the Student t-test (normal distribution) or the Wilcoxon rank-sum test (non-normal distribution) to compare numerical variables.

All statistical tests were two-tailed, and an a error of up to $5 \%$ was accepted to define the statistical significance of any results. Statistical analyses were performed using Stata MP 16.1 (StataCorp ${ }^{\circledR}$ )

\section{Results}

We included 141 patients in our final analysis, with a median age of 72 (IQR 68-79) years and a predominance of the male sex $(58 \% ; \mathrm{N}=82)$. We identified delirium at admission in 31 participants (22\%). Only $4(3 \%)$ participants had a baseline diagnosis of dementia. Patients with delirium also had a higher prevalence of other comorbidities. Table 1

Table 1

Baseline Characteristics

\begin{tabular}{|lll|}
\hline Variable & $\begin{array}{l}\text { Septic Patients } \\
(\mathbf{n = 9 5 )}\end{array}$ & $\begin{array}{l}\text { COVID-19 Patients } \\
(\mathbf{n}=46)\end{array}$ \\
\hline Median age (years) [IQR] & $73(69-80)$ & $70(66-76)$ \\
\hline Sex & & $26(57)$ \\
\hline Male, $\mathrm{n}(\%)$ & $56(59)$ & $20(43)$ \\
\hline Female, $\mathrm{n}(\%)$ & $39(41)$ & $1(2)$ \\
\hline Dementia, $\mathrm{n}(\%)$ & $3(3)$ & $33(72)$ \\
\hline Hypertension, $\mathrm{n}(\%)$ & $64(65)$ & $23(50)$ \\
\hline Diabetes, $\mathrm{n}(\%)$ & $41(43)$ & $7(15)$ \\
\hline Obesity, $\mathrm{n}(\%)$ & $1(1)$ & $19(41)$ \\
\hline SOFA $\geq 2, \mathrm{n}(\%)$ & $94(100)$ & $11(24)$ \\
\hline Delirium, $\mathrm{n}(\%)$ & $20(21)$ & \\
\hline
\end{tabular}

Intensive care admission and in-hospital mortality were different across groups, with unfavorable estimates in the delirium group. Although sepsis for COVID-19 has had more than $28 \%$ mortality, COVID19 was not associated with higher mortality in the elderly than sepsis for other etiologies $(p=0.15)$. Table 
Table 2

Association Between Delirium and Adverse Outcomes in Hospitalized Older Adults

\begin{tabular}{|c|c|c|c|c|c|c|}
\hline & \multicolumn{2}{|c|}{$\begin{array}{l}\text { Septic Patients } \\
(n=95)\end{array}$} & \multirow[t]{3}{*}{$p$} & \multirow{2}{*}{\multicolumn{2}{|c|}{$\begin{array}{l}\text { COVID-19 Patients } \\
(n=46) \\
\text { Delirium }\end{array}$}} & \multirow[t]{3}{*}{$p$} \\
\hline & \multicolumn{2}{|l|}{ Delirium } & & & & \\
\hline & Yes $(n=20)$ & No $(n=75)$ & & Yes $(n=11)$ & No $(n=35)$ & \\
\hline Death, n (\%) & $6(30)$ & $11(15)$ & 0.11 & $6(54)$ & $7(20)$ & 0.26 \\
\hline ICU admission, n (\%) & $5(25)$ & $21(28)$ & 0.79 & $4(36)$ & $16(46)$ & 0.92 \\
\hline
\end{tabular}

\section{Discussion}

This present study does not support elderly patients with COVID-19 have a higher risk for delirium at admission in emergency department when compared to patients hospitalized with sepsis for other etiologies.

Before the COVID-19 pandemics, delirium was a common presenting condition for older adults in the ED; however, two-thirds of cases went undetected. (14) Early COVID-19 studies have estimated rates of delirium about $30 \%$ in elderly hospitalized patients. (15) In a multicenter retrospective cohort study, $28 \%$ of 817 older patients with SARS-CoV-2 infection had delirium on arrival to the ED (8). The authors explained the high rate of delirium because they used a structured delirium assessment tool and the high prevalence of comorbid conditions in their study population. However, $30 \%$ of these patients had a prior diagnosis of cognitive impairment or dementia, the most critical risk factor for delirium. (16) (17)

The possible mechanisms behind the association between COVID-19 and delirium are also intriguing. Previous evidence suggests that SARS-CoV-2 may be a neurotropic virus and can cause direct brain damage by increasing demyelination, interleukin release, and blood-brain barrier permeability (18) (19) The main question was whether delirium in COVID-19 patients is caused by these neuroinflammatory pathways or by the direct action of the SARS-CoV-2 virus.

It is known that central nervous system inflammation participates in the pathogenesis of delirium; therefore, we compared a condition where systemic inflammation is also present.(19)

Despite possible distinct pathophysiological processes, sepsis-associated encephalopathy (SAE) is a diffuse brain dysfunction secondary to infection without overt CNS infection. (20) SAE presents itself in several degrees of severity and includes delirium.

Interestingly, in a prospective postmortem study of patients with sepsis, cerebral lesions were reported, which in one patient were compatible with multifocal necrotizing leukoencephalopathy and evidence of cerebral ischemia and hemorrhages. (21) Similar findings were observed in COVID-19 patients. (22) 
Therefore, until there is more evidence showing SARS-CoV-2 -related neuronal injury, we believe that Delirium associated with COVID-19 could be considered an SAE spectrum.

Our data also show the relevance of diagnosing delirium in these patients. In our cohort, Delirium at admission in Emergency Department was significantly associated with mortality during hospitalization ( $p$ $=0.007)$, which agrees with other authors.

This present study has some limitations. First, our study was performed in a single center dedicated to high-complexity medical care, and our results should be read with parsimony before being generalized to different populations. On the other hand, it is a strength because we reproduced the same delirium (14) assessment tool, and patients with COVID-19 and sepsis were hospitalized in similar conditions and treated by the same staff in a short time difference. Second, our Delirium incidence in septic patients was high, which does not allow us to prove our hypothesis with our previous sample size. Lastly, this was an Emergency Department study, and we did not do assessments regarding delirium duration and severity.

Despite these limitations, our study addresses two fundamental questions regarding delirium in COVID-19 patients. First, it suggests that the pathophysiology of delirium in COVID-19 patients may be related more to systemic inflammation than to the direct action of the SARS-CoV-2 virus in the central nervous system. Second, it points out the fundamental role of diagnosing delirium in ED. (23)

During the pandemics, many EDs are overcrowded, placing ED providers under enormous pressure to care for critically ill or injured patients simultaneously, care for patients boarding in the ED while awaiting an inpatient bed, and balancing other clinical duties, including other screening priorities, as a suicide risk and domestic violence. (24) Under these circumstances, we believe that demonstrating the association between delirium admission and in-hospital mortality remains important to aware ED providers to adopt and use delirium screening protocols. Nevertheless, this study showed vital data about delirium prevalence in elderly septic patients at admission to ED.

\section{Conclusion}

In summary, infection by SARS-COV-2 was not associated with higher delirium at emergency department admission. Furthermore, the prompt recognition of delirium is critical to ensure appropriate clinical care and prevent adverse outcomes in the elderly.

\section{List Of Abreviation}

CAM: Confusion Assessment Method

ED: Emergency Department

HCFMUSP: Hospital das Clínicas da Faculdade de Medicina da Universidade de São Paulo ICU: Intensive Care Unit 
RT-PCR: Real-time polymerase chain reaction

SOFA: Sequential Organ Failure Assessment

\section{Declarations}

Ethics approval and consent to participate:

The first protocol for this study was approved by the local Ethics Committee in Research, Comissão de Ética para Análise de Projetos de Pesquisa do HCFMUSP, at Hospital das Clínicas da Faculdade de Medicina da Universidade de São Paulo (Protocol number 77169716.2.0000.0068), and informed consent by writing was obtained from all patients or legal guardians prior to inclusion.

The second study protocol was approved by the local Research Ethics Committee, Comissão de Ética para Análise de Projetos de Pesquisa do HCFMUSP, at Hospital das Clínicas da Faculdade de Medicina da Universidade de São Paulo (protocol number 30417520.0.0000.0068). Written informed consent or verbal authorization from the patient or responsible family member was documented in the medical record to guarantee the patient's anonymity preservation.

Consent for publication

All authors contributed significantly to this work and have read and approved the submission of the manuscript. Also, this manuscript has not been published and is not being considered for publication elsewhere, in whole or in part, in any language.

Availability of data and material:

The data that support the findings of this study are available from the corresponding author, JCGA, upon reasonable request.

Competing interests

None

Funding

Fundação de Amparo à Pesquisa do Estado de São Paulo (FAPESP), Grants \#2020/04.738-8 and \#2016/14.566-4.

Authors' contributions

HPS, JCGA, FBGC, ADMV, and GS conceived the study, designed the trial, and obtained research funding. HPS and TA supervised the conduct of the trial and data collection. ADMV, IWA, and JC undertook recruitment of participating centers and patients and managed the data, including quality control. JCGA and LMGG provided statistical advice on study design and analyzed the data. All authors drafted the 
manuscript, and all authors contributed substantially to its revision. JCGA takes responsibility for the paper as a whole

Acknowledgements:

ED USP COVID Group 1: João Carlos Pereira Gomes, João Vitor Mahler Ferreira Oliveira, Amanda Dafne Zara, Gabriel Kenji Ito, Gustavo Henrique Mori, Isabelly Victoria SimoesMelchiori, Laura Fernandes Berto, Lucas de Mello Queiroz, Luiza Maria Goldstein Rubio, Marcia HarumyYoshikawa, Marco Antonio Rodrigues e Mello, Pollyana Karine Lopes dos Santos

\section{References}

1. Oliveira J. e Silva L, Berning MJ, Stanich JA, Gerberi DJ, Murad MH, Han JH, et al. Risk Factors for Delirium in Older Adults in the Emergency Department: A Systematic Review and Meta-Analysis. Ann Emerg Med [Internet]. 2021 Jun; Available from: https://linkinghub.elsevier.com/retrieve/pii/S0196064421001943

2. Barron EA, Holmes J. Delirium within the emergency care setting, occurrence and detection: A systematic review. Emerg Med J [Internet]. 2013 Apr;30(4):263-8. Available from: https://emj.bmj.com/lookup/doi/10.1136/emermed-2011-200586

3. Inouye SK, Westendorp RG, Saczynski JS. Delirium in elderly people. Lancet [Internet]. 2014 Mar;383(9920):911-22. Available from: https://linkinghub.elsevier.com/retrieve/pii/S0140673613606881

4. Suffoletto B, Miller T, Frisch A, Callaway C. Emergency physician recognition of delirium. Postgrad Med J [Internet]. 2013 Nov;89(1057):621-5. Available from: https://pmj.bmj.com/lookup/doi/10.1136/postgradmedj-2012-131608

5. Mao L, Jin H, Wang M, Hu Y, Chen S, He Q, et al. Neurologic Manifestations of Hospitalized Patients with Coronavirus Disease 2019 in Wuhan, China. JAMA Neurol. 2020;77(6):683-90.

6. Romero-Sánchez CM, Díaz-Maroto I, Fernández-Díaz E, Sánchez-Larsen Á, Layos-Romero A, GarcíaGarcía J, et al. Neurologic manifestations in hospitalized patients with COVID-19: The ALBACOVID registry. Neurology. 2020;95(8):e1060-70.

7. Romoli M, Jelcic I, Bernard-Valnet R, García Azorín D, Mancinelli L, Akhvlediani T, et al. A systematic review of neurological manifestations of SARS-CoV-2 infection: the devil is hidden in the details. Eur J Neurol. 2020;27(9):1712-26.

8. Kennedy M, Helfand BKI, Gou RY, Gartaganis SL, Webb M, Moccia JM, et al. Delirium in Older Patients With COVID-19 Presenting to the Emergency Department. JAMA Netw open [Internet]. 2020 Nov 19;3(11):e2029540. Available from: https://jamanetwork.com/journals/jamanetworkopen/fullarticle/2773106

9. Pun BT, Badenes R, Heras La Calle G, Orun OM, Chen W, Raman R, et al. Prevalence and risk factors for delirium in critically ill patients with COVID-19 (COVID-D): a multicentre cohort study. Lancet 
Respir Med [Internet]. 2021 Jan; Available from:

https://linkinghub.elsevier.com/retrieve/pii/S221326002030552X

10. Singer M, Deutschman CS, Seymour C, Shankar-Hari M, Annane D, Bauer M, et al. The third international consensus definitions for sepsis and septic shock (sepsis-3). JAMA - J Am Med Assoc [Internet]. 2016 Feb 23;315(8):801-10. Available from: http://jama.jamanetwork.com/article.aspx? doi=10.1001/jama.2016.0287

11. Harris PA, Taylor R, Minor BL, Elliott V, Fernandez M, O'Neal L, et al. The REDCap consortium: Building an international community of software platform partners. J Biomed Inform [Internet]. 2019 Jul;95:103208. Available from: https://linkinghub.elsevier.com/retrieve/pii/S1532046419301261

12. Inouye SK, Van Dyck CH, Alessi CA, Balkin S, Siegal AP, Horwitz RI. Clarifying confusion: The confusion assessment method: A new method for detection of delirium. Ann Intern Med. 1990;113(12):941-8.

13. Fabbri RMA, Moreira MA, Garrido R, Almeida OP. Validity and reliability of the portuguese version of the confusion assessment method (CAM) for the detection of delirium in the elderly. Arq Neuropsiquiatr. 2001;59(2 A):175-9.

14. Kennedy M, Enander RA, Tadiri SP, Wolfe RE, Shapiro NI, Marcantonio ER. Delirium Risk Prediction, Healthcare Use and Mortality of Elderly Adults in the Emergency Department. J Am Geriatr Soc [Internet]. 2014 Mar;62(3):462-9. Available from:

https://onlinelibrary.wiley.com/doi/10.1111/jgs.12692

15. Garcez FB, Aliberti MJR, Poco PCE, Hiratsuka M, Takahashi S de F, Coelho VA, et al. Delirium and Adverse Outcomes in Hospitalized Patients with COVID-19. J Am Geriatr Soc [Internet]. 2020 Nov 5;68(11):2440-6. Available from: https://onlinelibrary.wiley.com/doi/10.1111/jgs.16803

16. Ahmed S, Leurent B, Sampson EL. Risk factors for incident delirium among older people in acute hospital medical units: a systematic review and meta-analysis. Age Ageing [Internet]. 2014 May 1;43(3):326-33. Available from: https://academic.oup.com/ageing/articlelookup/doi/10.1093/ageing/afu022

17. Cecília de Melo S, Champs APS, Goulart RF, Malta DC, Passos VM de A. Dementias in Brazil: Increasing burden in the 2000-2016 period. Estimates from the Global Burden of Disease Study 2016. Arq Neuropsiquiatr [Internet]. 2020 Dec;78(12):762-71. Available from: http://www.scielo.br/scielo.php?script=sci_arttext\&pid=S0004-282X2020001200762\&tlng=en

18. Wu Y, Xu X, Chen Z, Duan J, Hashimoto K, Yang L, et al. Nervous system involvement after infection with COVID-19 and other coronaviruses. Brain Behav Immun [Internet]. 2020 Jul;87:18-22. Available from: https://linkinghub.elsevier.com/retrieve/pii/S0889159120303573

19. Maldonado JR. Delirium pathophysiology: An updated hypothesis of the etiology of acute brain failure. Int J Geriatr Psychiatry [Internet]. 2018 Nov;33(11):1428-57. Available from: http://doi.wiley.com/10.1002/gps.4823

20. Gofton TE, Young GB. Sepsis-associated encephalopathy. Nat Rev Neurol [Internet]. 2012 Oct 18;8(10):557-66. Available from: http://www.nature.com/articles/nrneurol.2012.183 
21. Sharshar T, Gray F, Poron F, Raphael JC, Gajdos P, Annane D. Multifocal necrotizing leukoencephalopathy in septic shock. Crit Care Med. 2002;30(10):2371-5.

22. Lee M-H, Perl DP, Nair G, Li W, Maric D, Murray H, et al. Microvascular Injury in the Brains of Patients with Covid-19. N Engl J Med. 2021;384(5):481-3.

23. Hullick C, Conway J, Higgins I, Hewitt J, Stewart B, Dilworth S, et al. An assistant workforce to improve screening rates and quality of care for older patients in the emergency department: Findings of a pre- post, mixed methods study. BMC Geriatr. 2018;18(1).

24. Morley C, Unwin M, Peterson GM, Stankovich J, Kinsman L. Emergency department crowding: A systematic review of causes, consequences and solutions. Bellolio F, editor. PLoS One [Internet]. 2018 Aug 30;13(8):e0203316. Available from: https://dx.plos.org/10.1371/journal.pone.0203316 